\title{
An Optimal Inspection Policy for Protection Devices of Electronic Equipment Using Delay Time Model
}

\author{
Jiang Xin-liang, Zhao Jian-min and Li Zhi-wei \\ Mechanical Engineering College, Shijiazhuang 050003, China \\ productive@126.com
}

\begin{abstract}
In order to reduce or eliminate the consequences of the electronic equipment malfunction, some important electronic equipment were installed protection devices. Failures of protection devices are of hidden feature, and can only be found by inspections. Protection device failing to system malfunctioning has a delay characteristic. According to this delay characteristic, an inspection policy was proposed based on delay time model. An inspection model was developed to minimize the average cost per unit time with constraint of availability. Based on the model, optimal inspection interval can be determined to meet the objective of the optimization model. Finally, an example proves that the model is effective and economical.
\end{abstract}

Keywords: electronic equipment; protection devices; inspection interval; delay time; hidden function failure

\section{Introduction}

Protective devices are widely used in electronic equipment, in order to avoid or reduce the loss caused by system function failure. But whether the protection device is failure for the operator is unknown, therefore these function failure of protective device is hidden [1]. If a failure of the protection device remains unknown in the system, significant loss may be caused once an accidence or failure happens to the system and the protection device is required to perform its protection function. Therefore, it is very important to detect the failures of protection devices. IN practice, the most effective way to detect the hidden function failure is to monitor or inspection its hidden failure, and takes timely measures to prevent the occurrence of function failure. Therefore, development of an optimal inspection policy of protection device is an important task for asset management of electronic equipment.

In recent years, a lot of researches have done to solve the problem of product inspection and repair interval decision-making. For example, in references [1-3], a variety of mathematical models represented by "time detection model" were developed; In order to solve the problem of maintenance decision of products with potential failure, Zhao $[4,5]$ developed a two-stage function inspection model; In order to solve the problem of inspection decision-making for systems with hidden or unrevealed failures, references [6-8] had done a lot of researches work. However, most of the above models are based on the assumption of inspection is perfect (all of the hidden functional failure can be detected), and most of these studies only consider the economy as the objective, obviously, it is not consistent with the actual situation. However, most of the above models are based on the assumption of inspection is perfect (all of the hidden functional failure can be detected), and most of these studies only consider the economy as the objective, obviously, it is not consistent with the actual situation. In addition, less work has been done to develop inspection polices in order to detect hidden failures of protection devices. Therefore in this paper, an inspection model was developed to optimize inspection interval, 
and imperfect inspection was taken into account which represented actual situation of system maintenance. System availability was used as the constraint, average cost per unit time as the optimization goal in the development of the inspection optimization model. We also analyses the impact of inspection intervals for maintenance costs and availability of unit time specially.

\section{Inspection Policy and Assumptions}

\subsection{Inspection Policy}

Usually, failures of protective devices are not observable for the operation crew of electronic equipment. If a failure of the device is not detected, it will cause significant loss in the case that a failure or event happens to the system which the device is to protect, Therefore, in order to prevent the multiple failures, the effective inspection tools and methods may be employed.

In general, the electronic equipment may have three states, including normal state, state of hidden functional failure (the protective device fails and no multiple failure occurs), and functional failure state. Figure 1 shows the ordering relations of three states.

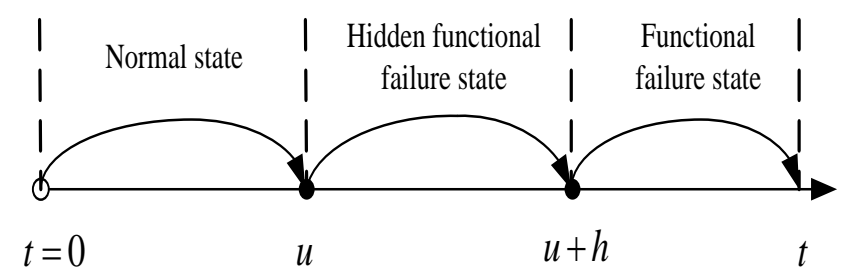

Figure 1. Process of Functional Failure

Normal state is the initial state of delivered equipment, and at this moment, the equipment is neither in hidden functional failure state nor in functional failure state. Hidden functional failure state is a kind of state that the protective device failed, but this failure has not triggered the equipment failure, in other word, the equipment can still work in a state the probability of functional failure is increased. Functional failure rate means the equipment cannot fulfill the specified function, and this state may be caused by protective device failure or by its own failure. We name the characteristics that functional failures happen after hidden functional failures as delay characteristics, and the time from when the hidden functional failure occurred to when the functional failure happened was called delay time. This concept used to describe the trend of hidden functional failure which share common features with PF curves was raised by professor Christer. Therefore, the delay time model proposes a feasible solution for inspection strategy of hidden functional failure, and this inspection strategy model and other inspection models have more than a little in common.

Summarizing the above analysis, the inspection strategy can be described as following:

(1)We study events and probabilities related to hidden functional failure and functional failure in one update cycle, which was considered as the $k+1$ th inspection interval, and the duration of each inspection is the same.

(2)The maintenance is conducted immediately if only the electronic equipment fails during inspection interval.

(3)If functional failure has not occurred during inspection interval but detected hidden functional failure, maintenance should also be conducted immediately to avoid the happening of multiple failure. 
(4)Giving that neither functional failure nor hidden functional failure is happened at inspection point, the current inspection is over, and the next inspection begins, which obeys the same inspection strategy.

\subsection{Basic Assumptions}

Owing to the complexity of field inspection, some assumptions were made to simplify the modeling procedure.

(1) The maintaining for functional failure and hidden functional failure can recover the equipment into normal state.

(2) The time of functional inspection and the time of maintenance are shorter than inspection period.

(3)The inspection is not perfect, the probability of finding hidden functional failure in inspection for once is $\delta$.

(4)The functional failure of electronic equipment triggered by inherent failure and by hidden functional failure are independent.

\section{Development of Model}

\subsection{Renewal Cycle of System}

We use $p(u)$ and $P(u)$ to denote the hidden failure density function and distribution function, $q(h)$ and $Q(h)$ to denote the delay time density function and distribution function. The inherent reliability of equipment is denoted by $R_{s}(t)$.

We analysis the events of happening of hidden failure and function failure in the $k+1$ th inspection and the probabilities of these events. In the time of $(k+1) T$, the events of finding hidden failure or function failure include:

Case 1: The hidden failures occurred before $k T$, and has not been detected before $k T$. The hidden failure has not become function failure, and is detected in $(k+1) T$.

The events that hidden failures occurred before $k T$ have many possibilities. The hidden failures may occur in $[0, T],[0,2 T] \cdots[(k-1) T, k T]$, and the delay time point $h$ is beyond $(k+1) T$.

Where, for the $i$ th hidden failure happened in $[(i-1) T, i T]$, the probability of detecting the hidden failure firstly in $(k+1) T$ is:

$$
\int_{(i-1) T}^{i T} \delta(1-\delta)^{k-i+1} R_{S}(u) p(u)\{1-Q[(k+1) T-u]\} d u
$$

Then, the probability of case 1 is:

$$
C_{1}(T, k)=\sum_{i=1}^{k} \int_{(i-1) T}^{i T} \delta(1-\delta)^{k-i+1} R_{S}(u) p(u)\{1-Q[(k+1) T-u]\} d u
$$

Case 2: The hidden failure occurred after $k T$, and has not become function failure. The hidden failure was been detected in $(k+1) T$. The probability of case 2 is:

$$
C_{2}(T, k)=\int_{k T}^{(k+1) T} \delta R_{S}(u) p(u)\{1-Q[(k+1) T-u]\} d u
$$

Case 3: The hidden failure has been occurred before $k T$, and did not been detected at every inspection before $k T$. The function failure was happened in $[k T,(k+1) T]$. 
The same to case 1, the events that hidden failures happened before $k T$ had many possibilities. The hidden failure may occur in $[0, T] 、[T, 2 T] \cdots[(k-1) T, k T]$. The probability of case 3 is:

$$
C_{3}(T, k)=\sum_{i=1}^{k}\left\{\int_{(i-1) T}^{i T}(1-\delta)^{k-i+1} R_{S}(u) p(u) \int_{k T-u}^{(k+1) T-u} q(h) d u d h\right\}
$$

Case 4: The hidden failure happened after $k T$, and became function failure in $[k T,(k+1) T]$. The probability of case 4 is:

$$
C_{4}(T, k)=\int_{k T}^{(k+1) T} R_{s}(u) p(u) \int_{0}^{(k+1) T-u} q(h) d h d u
$$

Case 5: It is function failure that happened in $[k T,(k+1) T]$ rather than hidden failure.

The main factor of this event is the inherent reliability. The failure density function influenced by inherent reliability is $-R_{S}^{\prime}(t)$. The probability of the event that hidden failure did not occur is $1-P(t)$. Then, the probability of case 5 is:

$$
C_{5}(T, k)=\int_{k T}^{(k+1) T}-R_{S}^{\prime}(t)[1-P(t)] d t
$$

It is defined the time period that starts from the equipment begins to work, ends when function failure happens or hidden failure is been discovered as renewal cycle. We use $T_{k}$ to denote the replacement time interval when the events of replacement happen in $[k T,(k+1) T]$. As for the events that hidden failures are discovered in $(k+1) T$ ( see case 1 and case 2), the replacement time interval is: $T_{k}=(k+1) T$.

As for the events that function failures are discovered in $[k T,(k+1) T]$ (see case 3、 4 and 5), the replacement time interval is: $T_{k}=u+h$.

Therefore, the expectation of replacement interval can be given by:

$$
\begin{aligned}
& E_{R}(T)=\sum_{k=0}^{\infty} E\left(\mathrm{~T}_{k}\right) \\
& =\sum_{k=0}^{\infty}(k+1) T \sum_{i=1}^{k} \int_{(i-1) T}^{i T} \delta(1-\delta)^{k-i+1} R_{S}(u) \mathrm{p}(u)\{1-Q[(k+1) T-u]\} d u \\
& +\sum_{k=0}^{\infty}(k+1) T \int_{k T}^{(k+1) T} \delta R_{S}(u) \mathrm{p}(u)\{1-Q[(k+1) T-u]\} d u \\
& +\sum_{k=0}^{\infty} \sum_{i=1}^{k} \int_{(i-1) T}^{i T} \int_{k T-u}^{(k+1) T-u}(u+h) \delta(1-\delta)^{k-i+1} R_{S}(u) \mathrm{p}(u) d h d u \\
& +\sum_{k=0}^{\infty} \int_{k T}^{(k+1) T} \int_{0}^{(k+1) T-u}(u+h) R_{S}(u) p(u) \mathrm{q}(h) d h d u \\
& +\sum_{k=0}^{\infty} \int_{k T}^{(k+1) T}-t R_{S}^{\prime}(t)[1-P(t)] d t
\end{aligned}
$$

\subsection{The Analysis of Costs}

As is similar with latent failure inspection, the main aim of hidden failure inspection is to find the hidden failure and repair it in time so as to decrease the loss caused by function failure. The costs include three parts in this inspection policy:

1) The function failure happened before an inspection, and was repaired. The cost of this condition spends very much.

2) The hidden failure was been detected by an inspection, and was restored to a state as new. The maintenance can be arranged during equipment does not work. In this case, the loss is much less than the loss caused by function failure. 
3) No hidden failure is detected by the inspection. The cost is only inspection fee.

Let $d_{i R}$ to denote the inspection fee, and $d_{a R}$ denote the cost of function failure and repair fee, $d_{b R}$ denote the cost of hidden failure and repair fee. In the following, the three kinds of costs are analysed respectively.

The probability of conducting $n$ times inspections in one renewal cycle is:

$$
C_{n}=C_{3}(T, n)+C_{4}(T, n)+C_{1}(T, n-1)+C_{2}(T, n-1)
$$

As a consequence, the mean inspection number in one renewal cycle is $\sum_{n=1}^{\infty} n C_{n}$. The mean inspection fee in one renewal cycle is $d_{i} \sum_{n=1}^{\infty} n C_{n}$. The probability of the happening of function failure in one renewal cycle is :

$$
A_{R}(T)=\sum_{n=0}^{\infty}\left[C_{3}(T, n)+C_{4}(T, n)+C_{5}(T, n)\right]
$$

The probability that hidden failure can be discovered by inspection in one renewal cycle is:

$$
B_{R}(T)=\sum_{n=0}^{\infty}\left[C_{1}(T, n)+C_{2}(T, n)\right]
$$

Both function failure and hidden failure induce replacement of components, so the mean cost in one renewal cycle is:

$$
d_{i} \sum_{n=1}^{\infty} n C_{n}+d_{a R} A_{R}(T)+d_{b R} B(T)
$$

According to renewal cycle theory, the mean cost per unit of time is given by:

$$
C_{R}(T)=\frac{d_{i} \sum_{n=1}^{\infty} n C_{n}+d_{a R} A_{R}(T)+d_{b R} B(T)}{E_{R}(T)}
$$

Using Eq. (10), the optimal inspection interval, T, can be obtained by doing derivation to $C_{R}(T)$, which will minimize the mean cost per unit of time.

\subsection{The Analysis of Availability}

System availability is the ability of a product or service to be ready for use when the customer wants to use it. It can be expressed as the ratio of working time to total time in a renewal cycle. The derivation process of availability model is similar with the cost model, and time is the focus of analysis.

Let $T_{i}$ denote the time for an inspection, and $T_{a}$ denote the time for function failure repair, $T_{b}$ denote he time for hidden function failure repair.

According to the derivation of the cost model, we have:

The accumulative inspection time in one renewal cycle is: $T_{i} \sum_{n=1}^{\infty} n C_{n}$; The accumulative time for function failure repair in one renewal cycle is: $T_{a} A_{R}(T)$; The accumulative time for hidden function failure repair in one renewal cycle is: $T_{b} B_{R}(T)$.

So, the accumulated down time in one renewal cycle is: 


$$
E D=T_{i} \sum_{n=1}^{\infty} n P_{n}+T_{a} A_{R}(T)+T_{b} B_{R}(T)
$$

According to the theory of renewal process, the average availability in one renewal cycle can be expressed as:

$$
A_{a v}(T)=\frac{E_{R}(T)-E D}{E_{R}(T)}
$$

\subsection{The Optimization Model of Inspection}

In general, the most worthwhile optimization goal is the system availability meet requirement and at the same time the average cost per unit time minimum.

Thus, in this paper, an inspection optimization model was developed, where $\mathrm{T}$ is the optimization variable, system availability is the constraint function, and let average cost per unit time minimum as the optimization goal.

$$
\begin{array}{ll}
\min & C_{R}(\mathrm{~T})=\frac{d_{i} \sum_{n=1}^{\infty} n C_{n}+d_{a R} A_{R}(\mathrm{~T})+\mathrm{d}_{\mathrm{bR}} B_{R}(\mathrm{~T})}{E_{R}(\mathrm{~T})} \\
\text { s.t. } & A_{a v}(\mathrm{~T})=\frac{E_{R}(\mathrm{~T})-\mathrm{ED}}{E_{R}(\mathrm{~T})} \geq A_{0}
\end{array}
$$

\section{Case Study}

In order to reduce or eliminate the consequences of the failure of low-voltage three-phase motor, a multifunctional protection device is often installed. It can provide full protection for the various faults of the low-voltage three-phase motor, and improve the utilization and extend the service life of the motor. The data provided by the supplier show that, the mean time between failure of the lowvoltage three-phase motor is 300 days.

Each inspection cost of the protection device is about 20 Yuan, and takes about 0.5 working days; the costs of each maintenance and lost are about 2000Yuan, and the time to repair the failure take about 2 working days; the cost of maintenance of protection device is about 100 Yuan, and the time to repair the failure of protection device take about 1 working days. We assumed the availability requirements of the equipment is $A_{0} \geq 0.95$.

Below, we according to the above optimization model to solve the case.

\subsection{The Cost Model}

First, using the proposed models, we discuss the influence of value of $\delta$ to the inspection interval $T$ for which average cost per unit time is minimum. The case of $\delta=0.5 、 0.7 、 0.9$ is considered, and the average cost per unit time curves over inspection-interval $T$ can be obtained by use the MATLAB software, as shown in Figure 2. 


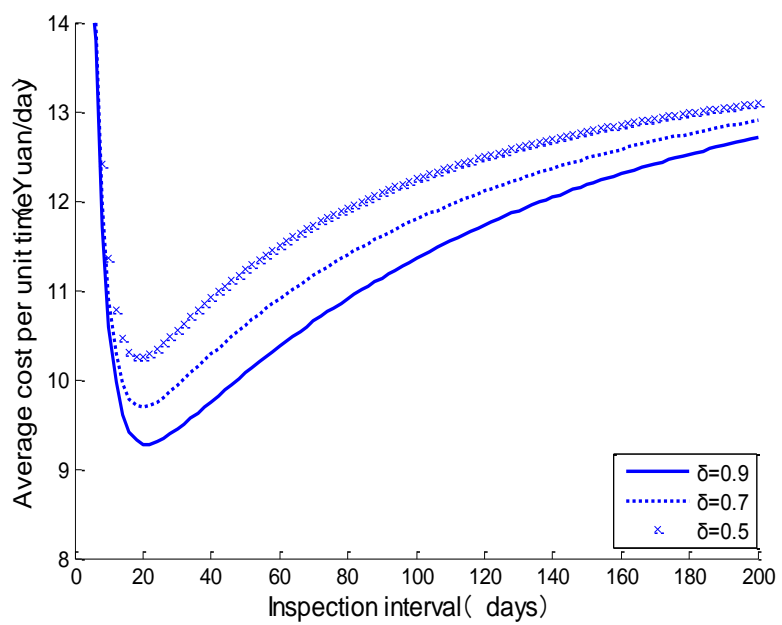

\section{Figure 2. The Relationship between Inspection-interval and the Average Cost per Unit Time}

As shown in Figure 2, no matter which value of $\delta$ is, there should be a corresponding lowest point in the cost curve, and this point is the inspection interval of the lowest rate of cost. Furthermore, it also can be seen from Figure 3, other things being equal, if the inspection rate of failure $\delta$ decreases, the rate of maintenance cost per unit time will increase. Instead, the corresponding inspection-interval will decrease. This is consistent with the actual situation of maintenance. Therefore, it is a good way to save the maintenance costs by improving the inspection rate of hidden function failure.

Using the Eq.10, the lowest average cost per unit time can be obtained for each case of imperfect inspection, as shown in Table 1:

Table 1. The Average Cost per Unit Time under Different Value of $\delta$

\begin{tabular}{ccc}
\hline$\delta$ & $T$ (days) & costs (Yuan/day) \\
\hline 0.5 & 20 & 10.26 \\
0.7 & 21 & 9.69 \\
0.9 & 22 & 9.28 \\
\hline
\end{tabular}

Below, we discuss the relationship between inspection-interval and single cost. We assume other things being equal, and let $\delta=0.9$, then the relationship between inspection-interval and single cost can be obtained by solving the above model, as shown in Figure 3: 


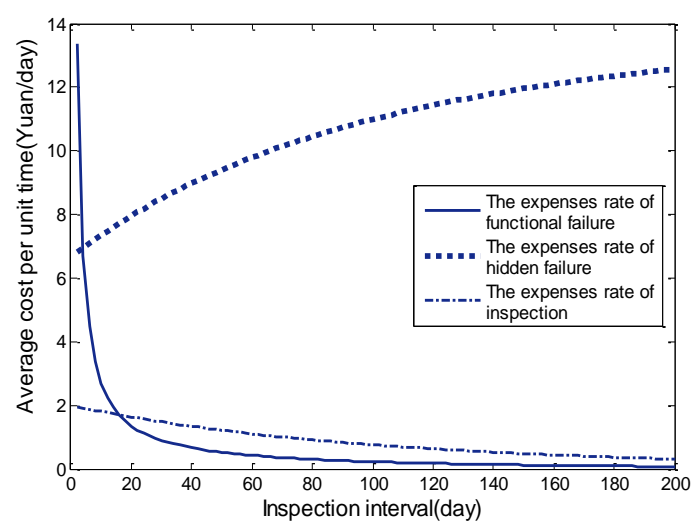

Figure 3. The Relationship between Inspection-interval and Single Cost

As shown in Figure 3, the maintenance cost of hidden function failure per unit time and the inspection cost per unit time will decrease with the inspection-interval increase, this is because in other costs under the condition of invariable, the number of inspection and maintenance costs of hidden function failure per unit time will decrease with the inspection-interval increase in length, and the costs of the corresponding can also reduce. But, on the other hand, the costs of maintenance and lost by function failure will gradually increase with the inspection-interval increase in length, this is because the inspection interval is too long to increase the probability of function failure, so the costs of maintenance caused by function failure will also increase, and this is consistent with the actual situation.

\subsection{The Availability Model}

According to the derivation of Eq.12 we can see, there is a close relationship between the system availability and total downtime of a replacement cycle. Below, we discuss the relationship between the total down and the inspection-interval.

It is assumed that the value of $\delta=0.5 、 0.7 、 0.9$, and all other parameters and conditions remain unchanged. Then, we can obtain the relationship between the replacement-cycle and inspection-interval by calculating Eq.6, as shown in Figure 4:

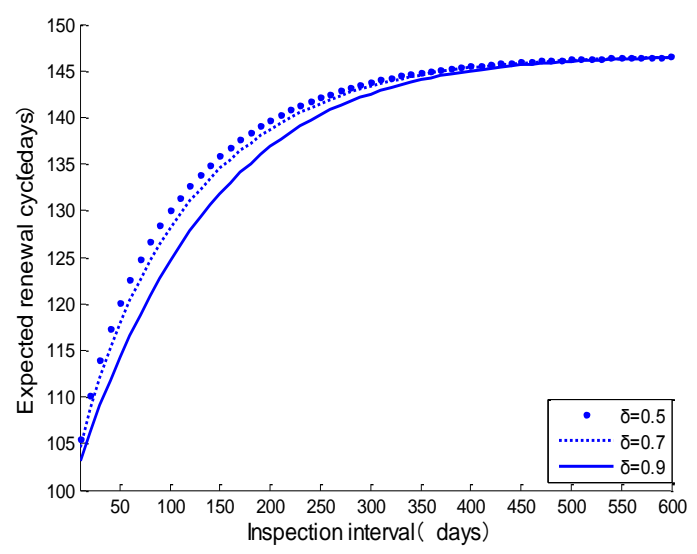
Figure 4. The Relationship between Expected Renewal-cycle and
Inspection-interval

It is can be seen from Figure 4, when the inspection-interval was the same, the higher the detection rate of hidden function failure, the shorter the expected renewal-cycle. This 
is because more lower the failure detection rate, more less the renewal times per unit time, this is match with the actual situation of maintenance.

Similarly, we can obtain the relationship between inspection-interval and total downtime of a renewal-cycle by calculating Eq.11, as shown in Figure 5:

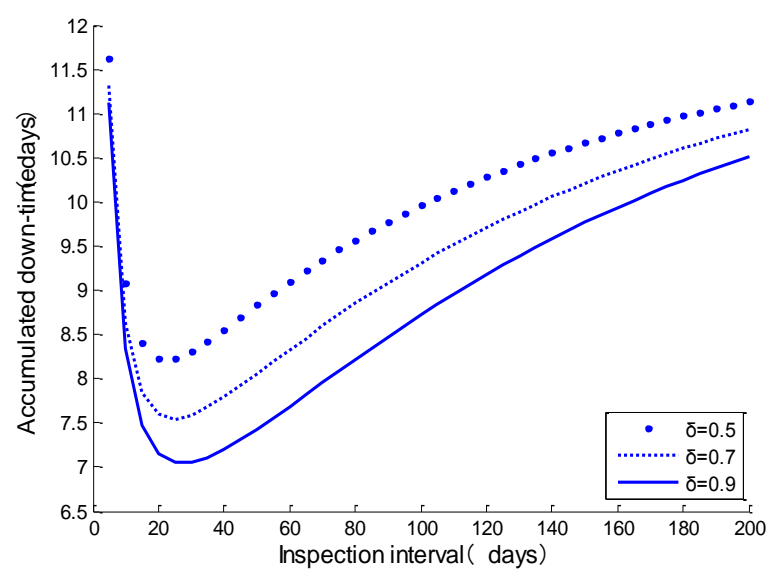

Figure 5. The Relationship between Total Downtime and Inspection-interval

As shown in Figure 5, there is an optimal inspection interval, which makes the cumulative downtime minimal in a single renewal cycle. Therefore, in the case of other conditions unchanged, if the inspection accuracy is reduced, the accumulation downtime of a single updating cycle will increase, and the corresponding optimal inspection interval will become shorter. Decreasing in accuracy of inspection will result in the hidden failure being not detected in time, thereby increasing the probability of equipment failure, and the corresponding failure repair time will be extended.

In above, we analyzed the relationship between the expectation of inspection interval and renewal cycle and the total downtime in a single renewal cycle. Next, we will analyze the relationship between the inspection interval and expected availability. Using MATLAB the solution of Eq.12 can be calculated, and we can obtain the trend of system expected availability changing with the interval, as shown in Figure 6:

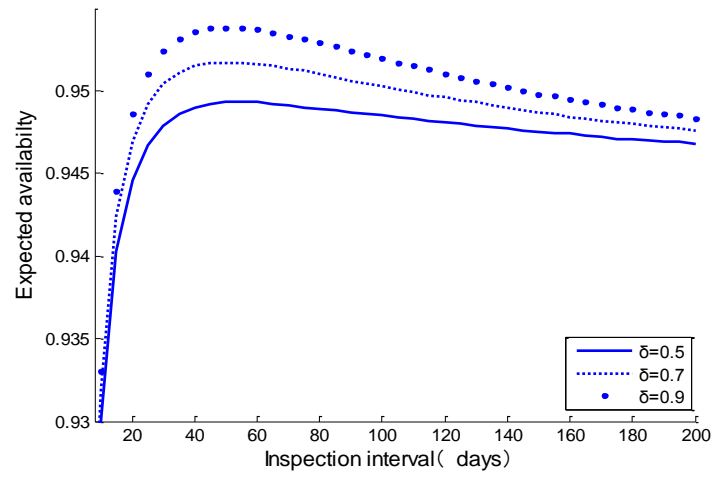

Figure 6. The Relationship between Expected Availability and Inspectioninterval

As shown in Figure 6, there is an optimal inspection cycle to make the maximum availability, and if the other things being equal, the higher the failure detection rate, the higher the system availability, this is match with the actual situation of maintenance too.

We can obtain the optimal inspection-interval in different value of $\delta$ after derivation Eg.12. As shown in Table.2:

Table 2. Maximum Availability in Different Value of $\delta$ 


\begin{tabular}{lcc}
\hline$\delta$ & $T$ (Days) & Availability \\
\hline 0.5 & 51.21 & 0.9493 \\
0.7 & 50.35 & 0.9517 \\
0.9 & 49.68 & 0.9538 \\
\hline
\end{tabular}

\subsection{The Optimization Model}

It is assumed the value of $\delta$ is 0.9. Computational results with the Eq.13 show that if the inspection-interval $T \in[23.62,146.76]$ days, the system availability $\mathrm{A}_{\mathrm{av}} \geq 0.95$ accord with the requirement. We did the cost analysis during that time frame and found when the inspection-interval $T=23.62$ days, the minimum cost per unit time is 9.30 Yuan.

\section{Conclusions}

Because of the characteristic of delay and hidden of failure of some protection device of electronic equipment, a functional inspection policy was proposed and the availability mathematical model and cost rate mathematical model of the protection device are provided in accordance with the inspection policy. An optimal model was constructed to calculate the inspection intervals under the policy, which aims to minimize cost rate, and is subject to system availability. Furthermore, the algorithm of the optimal inspection model is also provided, through which the values of optimal intervals can be obtained. Finally, an example is illustrated to prove the validity of the strategy and models above.

\section{References}

[1] C. Jing, Z. Hongfu and W. Huawei, “Optimal Inspection Model Using Delay-Time Concept”, Journal of Applied Sciences, vol. 25, no. 4, (2007), pp. 397-401.

[2] C. Jinhua and C. Kan, "A Mathematical Introduction to Reliability", Bei Jing: Higher Education Press, (2005).

[3] W. Wang and A. H. Christer, "Solution Algorithm for a Non-homogeneous Multi-component Inspection Model", Computers and Operations Research, vol. 30, no. 1, (2003), pp. 19-34.

[4] Z. Jianmin and W. Yunfeng, "Astudy on Modeling Functional Inspection at Two Stages", Systems Engineering and Electronics, vol. 22, no. 12, (2000), pp. 49-51.

[5] Z. Jianmin and B. Jinda, "Optimal functional Inspection Policy with Considering Delay of Repair", Systems Engineering and Electronics, vol. 31, no. 5, (2009), pp. 1255-1258.

[6] T. Tang, D. Lin, D. Banjivic and A. K. S. Jardine, "Availability of A System subject to Hidden Failure Inspected Atconstant Intervals with Non-negligible Downtime Due to Inspection and Downtime Due to Repair/Replacement", Journal of Statistical Planning and Inference, vol. 143, (2013), pp. 176-185.

[7] J. Chen, J. S. Thorp and I. Dobson, "Cascading dynamics and mitigation assessment in power system disturbances via a hidden failure model", Electrical Power and Energy Systems, vol. 27, (2005), pp. 318-326.

[8] K. Bae and J. S. Thorp, “A stochastic study of hidden failures in power system protection”, Decision Support Systems, vol. 24, (1999), no. 259-268.

[9] A. H. Christer and W. H. Waller, "Reducing Production Downtime Using Delay Time Analysis", Journal of the Operational Research Society, vol. 35, no. 6, (1984), pp. 499-512. 